ORIGINAL

\title{
SATISFACCION LABORAL DE LOS PROFESIONALES DE ATENCIÓN PRIMARIA DEL ÁREA 10 DEL INSALUD DE MADRID
}

\author{
María Isabel Fernández San Martín (1), Antonio Moinelo Camporro (2), Adela Villanueva \\ Guerra (1), Cristina Andrade Rosa (4), Montserrat Rivera Teijido (3), Jose María Gómez Ocaña \\ (4) y Olga Parrilla Ulloa (5) \\ (1) Dirección Atención Primaria del Área sanitaria 10 del Insalud. Madrid. \\ (2) Comité Salud Laboral del Área sanitaria 10 del Insalud. Madrid. \\ (3) Equipo de Atención Primaria del centro de Salud Pintores. Área sanitaria 10 del Insalud. Madrid. \\ (4) Equipo de Atención Primaria del centro de Salud El Greco. Área sanitaria 10 del Insalud. Madrid. \\ (5) Equipo de Atención Primaria del centro de Salud Isabel II/I. Área sanitaria 10 del Insalud. Madrid. El Greco I. Área...
}

\section{RESUMEN}

Fundamento: La implantación de un modelo de calidad total requiere conocer la satisfacción laboral de los trabajadores. Pretendemos averiguar el nivel de satisfacción global y de cada uno de los componentes en la Atención Primaria del Área 10.

Métodos: Estudio descriptivo transversal realizado en el Area 10 del Insalud de Madrid, Atención Primaria. Trabajan en este Área 668 profesionales sanitarios y no sanitarios. Se utiliza el cuestionario de satisfacción Font Roja, adaptado por J. Aranaz, que mide la satisfacción laboral a través de una escala de 1 (menor grado de satisfacción) a 5 (mayor grado de satisfacción).

Resultados: Respondió el cuestionario un $80 \%$ de la población. La puntuación media de la satisfacción es de 3,12 (Error Típico $\mathrm{ET}=0,017$ ). Las dimensiones menos valoradas han sido la tensión relacionada con el trabajo, con una media de $2,30(\mathrm{ET}=0,041)$ y la promoción profesional con una media de 2,42 ( $E T=0,038)$. La dimensión más valorada es la competencia profesional con una media de 4,01 $(\mathrm{ET}=0,058)$.

Conclusiones: Los profesionales se encuentran medianamente satisfechos en su trabajo. Comparando este estudio con el realizado en este Área en 1992, concluimos que las dimensiones tensión laboral y promoción profesional siguen siendo las peor valoradas, lo que debería de activar medidas correctoras por parte de los equipos directivos.

Palabras clave: Satisfacción laboral. Profesionales sanitarios. Atención primaria. Control de calidad.

Correspondencia:

María Isabel Fernández San Martín

Dirección Atención Primaria. Area 10

Avda Juan de la Cierva s/n. Getafe.

28902 Madrid.

Correo electrónico: avillanueva@gapm10-insalud.es

\section{ABSTRACT}

\section{Job Satisfaction among Primary Care Professionals in Area 10 Insalud, Madrid}

Background: The implementation of a total quality model requires ascertaining the degree of employee job satisfaction. Our aim is to ascertain the overall degree of satisfaction and determine its components employed in Primary Care District No. 10.

Methods: Descriptive cross-sectional study conducted in Spanish National Health Institute Primary Health Care District No. 10 in Madrid. A total of 668 health care and non health care professionals are employed in this District. A Font Rojas satisfaction questionnaire adapted by J. Aranaz was used to gauge the degree of job satisfaction on a $1-5$ scale ( 1 being the lowest degree of satisfaction and 5 the highest).

Results: Eighty percent of the population answered the questionnaire. The average satisfaction mark was 3.12 (Standard Error SE=0.017). The aspects ranked the lowest were the stress involved in the job, averages 2.30 ( $\mathrm{SE}=0.041$ ) and professional promotion an average of 2.42 $(\mathrm{SE}=0.038)$. The aspect valued most highly was that of professional skill, averaging $4.01(\mathrm{SE}=0.058)$.

Conclusions: These professional are satisfied to an average degree with their work. On comparing this study with one conducted in this same District in 1992, we have drawn the conclusion that the aspects of work-related stress and professional promotion continue to be those ranked the lowest, which should lead to corrective measures being taken on the part of the management teams.

Key Words: Job satisfaction. Health care professionals. Primary Care. Quality Control. 


\section{INTRODUCCION}

La satisfacción de los profesionales de las instituciones sanitarias es un clemento que forma parte de la definición de la calidad de la atención prestada. Aunque se cuestione su relación directa sobre la calidad de la atención al cliente externo ${ }^{1}$, nadie discute que al medir la satisfacción laboral se está comprobando la calidad de la organización y de sus servicios internos.

El análisis global de la satisfacción es un indicador de poca utilidad para la puesta en marcha de medidas correctoras y seguimiento de dichas medidas. Los indicadores basados en cada una de las dimensiones que conforman la satisfacción laboral pueden ser más sensibles a los cambios introducidos en la organización de personal. Por tal motivo, es interesante utilizar cuestionarios que puedan analizar las dimensiones de la satisfacción laboral.

En nuestra Area Sanitaria se ha realizado un estudio de satisfacción laboral, como parte de uno más amplio de valoración de las condiciones de trabajo. Se quería comprobar, por una parte, la relación entre las condiciones laborales y ła satisfacción y, por otro lado, medir los cambios producidos en estos aspectos desde 1992, fecha en la que se realizó en esta misma Area una investigación similar ${ }^{2}$.

El presente estudio persigue el objetivo de conocer el nivel de satisfacción global y de cada uno de los componentes de la misma, en los trabajadores de Atención Primaria del Área 10 del Insalud de Madrid. Las dimensiones que se analizan son las siguientes: exceso o presión de trabajo, promoción profesional, satisfacción laboral, monotonía laboral, relaciones entre profesionales, competencia profesional, tensión relacionada con el trabajo y relaciones interpersonales entre compañeros.

\section{MATERIAL Y METODOS}

Estudio descriptivo transversal en el que se ha estudiado a todos los trabajadores de
Atención Primaria del Área 10 del Insalud de Madrid. Son 16 equipos y tres servicios de urgencias ubicados en 12 centros de salud. Se ha considerado como criterio de selección estar en activo en el momento del estudio y haber trabajado al menos 60 días en los últimos tres años. Con este criterio se quería incluir a aquellos profesionales que, siendo suplentes, conozcan las condiciones de trabajo existentes en el Area, y se excluyó a los estudiantes de enfermería en prácticas, ya que rotan menos de 60 días por los centros de salud. En este trabajo también se han excluido a los trabajadores sociales (4) y a los informáticos (2), ya que son un colectivo muy reducido y, debido a sus condiciones laborales, no parece razonable su agrupación en otras categorías.

En el momento de iniciar el estudio, segundo trimestre de 1997, la población que reunía los criterios anteriores era de 668 trabajadores.

Se ha utilizado el cuestionario Font Roja, adaptado por J. Aranaz ${ }^{3}$ de otro instrumento utilizado en el Tecumseh Community Health Study ${ }^{4}$, Este cuestionario mide la satisfacción general en el trabajo; consta de 24 preguntas y ha sido utilizado en el medio hospitalario. El análisis factorial de dicho estudio definió 9 factores como componentes de la satisfacción, que explican el $69,12 \%$ del total de la varianza. Posteriormente se realizó un estudio con el mismo cuestionario añadiendo cinco preguntas para los trabajadores de atención primaria del Area 10. Mediante el análisis factorial se describieron 8 dimensiones que explicaron el $58 \%$ de la varianza total ${ }^{2}$, Cada pregunta es valorada mediante una escala de Likert de $1-5$, en el sentido de menor a mayor grado de satisfacción. El punto 3 se toma como un grado medio de satisfacción.

También se han recogido variables universales (edad y sexo), variables que permiten valorar los criterios de selección (antigüedad en la empresa, lugar de trabajo), y otras variables independientes presumiblemente relacio- 
nadas con la satisfacción (tipo de contrato y categoría profesional). Se realizó un agrupamiento de las categorías profesionales debido al reducido número de profesionales de alguna de ellas que trabajan en el Area, valorando la similitud de tareas a realizar:

- Facultativos: médicos generales, pediatras, odontólogos, y médicos internos residentes (MIR) de Medicina Familiar y Comunitaria.

- Enfermería: enfermeras, matronas, y auxiliares enfermería.

- Personal no sanitario: administrativos, auxiliares administrativos, celadores y conductores.

- Empresas de servicios: personal de limpieza, de mantenimiento y vigilantes.

El trabajo de campo lo realizó el equipo investigador (formado por la responsable de salud laboral, un integrante del Comité de salud laboral, la técnica de salud pública y cuatro MIR), acudiendo a cada uno de los centros del Area para presentar los objetivos del estudio y explicar las instrucciones de cumplimentación del cuestionario a los profesionales, en el cual no iba a figurar el nombre del trabajador. No obstante, el equipo investigador se comprometía a guardar la confidencialidad de los datos y a presentar los resultados de forma agrupada. Los coordinadores de los equipos recogieron las encuestas y los remitieron por correo interno a la Gerencia de Atención Primaria. El plazo de contestación fue de dos semanas, finalizadas las cuales se remitió una carta dirigida a cada trabajador, recordándole la importancia de la contestación. Cuando el nivel de respuestas en algún centro era inferior al $80 \%$ se llamaba insistiendo en la importancia de la respuesta, dando un nuevo plazo de una semana más.

Se calculó la media y la mediana de cada una de las dimensiones de la escala para el global de la muestra, y los intervalos de confianza al $95 \%$; se compararon las puntuaciones de cada dimensión con las variables independientes mediante métodos estadísticos no paramétricos: Mann-Whitney y Kruskal-Wallis. Estos métodos se utilizan debido a que las dimensiones de la calidad no siguen una distribución normal y al reducido número de personas que se agrupan en alguna categoría.

\section{RESULTADOS}

El total de trabajadores que contestó el cuestionario es de 512, lo que representa el $80 \%$ de la plantilla. El porcentaje de respuesta ha sido superior en personal no sanitario $(84,0 \%)$ y en el de las empresas de servicios $(83,8 \%)$ en relación al personal sanitario $(75,1 \%$ facultativos y $75,7 \%$ enfermeras). El perfil de los profesionales es el siguiente: género femenino $(70,1 \%)$, estudios universitarios $(72,1 \%)$ y media de edad de 37 años (tabla 1). El 43\% tiene contrato en propiedad y similar porcentaje es personal interino. Los años trabajados en atención primaria son ocho, de media.

La puntuación media de la satisfacción laboral (tabla 2) es de 3,12 (IC.95\%:3,093,15 ). Las dimensiones menos valoradas (menor satisfacción) han sido la tensión relacionada con el trabajo, con una media de $2, .30\left(\mathrm{IC}_{95 \%}: 2,22-2,38\right)$ y la promoción profesional con una media de 2,42 (IC $_{95 \%}: 2,35$ 2,49 ). La dimensión más valorada es la competencia profesional (media: 4,01; IC I5\%: $_{\text {: }}$ : $3,90-4,12$ ). Se ha cuantificado por separado una pregunta específica sobre el grado de satisfacción en el trabajo, obteniéndose que un $25,8 \%$ de los trabajadores se encuentra poco o nada satisfecho.

Existen diferencias significativas entre la valoración que hacen las diferentes categorías de la mayoría de las dimensiones (tabla 3). Los facultativos son los que puntúan mas bajo el exceso de trabajo, 2,36 
Tabla 1

Perfil de los profesionales que han contestado

\begin{tabular}{|c|c|c|}
\hline & Frecuencia & $\%$ \\
\hline \multicolumn{3}{|l|}{ SEXO } \\
\hline Hombre & 150 & 29,9 \\
\hline Mujer & 351 & 70,1 \\
\hline \multicolumn{3}{|l|}{ ESTUDIOS } \\
\hline Ninguno o primarios sin acabar & 7 & 1,4 \\
\hline Graduado escolar/ E. primarios/ B. elemental & 50 & 9,9 \\
\hline BUP/B. superior/FP & 83 & 16,5 \\
\hline Universitarios medios & 150 & 29,8 \\
\hline Universitarios superiores & 213 & 42,3 \\
\hline \multicolumn{3}{|l|}{ CATEGORIA PROFESIONAL } \\
\hline Facultativos & 172 & 34,1 \\
\hline Enfermería & 159 & 31,6 \\
\hline Personal no sanitario & 142 & 28,2 \\
\hline Empresas de servicios & 31 & 6,2 \\
\hline \multicolumn{3}{|l|}{ TURNO DE TRABAJO: } \\
\hline Mañana & 233 & 46,3 \\
\hline Tarde & 192 & 38,2 \\
\hline Otros (dos turnos, noches, etc) & 78 & 15,5 \\
\hline \multicolumn{3}{|l|}{ TIPO DE CONTRATO: } \\
\hline Propiedad & 215 & 43,4 \\
\hline Interinidad & 205 & 41,4 \\
\hline Otros (sustitutos, tiempo parcial) & 75 & 15,2 \\
\hline \multirow{2}{*}{ EDAD } & Media & Desviación estándar \\
\hline & 37,00 & 8,27 \\
\hline TIEMPO TRABAJADO EN A.P. (años): & 8,16 & 5,18 \\
\hline
\end{tabular}

$\left(\mathrm{IC}_{95 \%}: 2,24-2,48\right)$, la tensión relacionada con el trabajo, 2,05 ( $\left.\mathrm{IC}_{95 \%}: 1,92-2,18\right)$, y las rela-

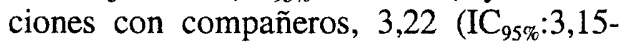
$3,29)$; la enfermería tiene la puntuación más elevada en el total de la escala de satisfacción, 3,20 ( $\left.\mathrm{IC}_{95 \%}: 3,14-3,26\right)$; el personal no sanitario tiene la media de puntuación más baja en la promoción profesional, 2,26 (IC ${ }_{95 \%}: 2,13-2,39$ ), en comparación con los otros estamentos. El personal de empresas es el que valora más negativamente las di- mensiones exceso de trabajo, 2,31 ( $\mathrm{IC}_{95 \%}$ : 2,07-2,55), satisfacción laboral, 2,89 ( $\mathrm{IC}_{95 \%}$ : 2,48-3,30), y monotonía laboral, 2,73 $\left(\mathrm{IC}_{95 \%}: 2,49-2, .97\right)$.

Analizando el nivel de satisfacción según el lugar de trabajo (tabla 4) se aprecia que los Servicios de Urgencias puntúan por debajo de los Equipos de Atención Primaria en las siguientes dimensiones: promoción profesional $\left(2,02, \mathrm{IC}_{95 \%}: 1,72-2,31\right.$, frente a 
Tabla 2

Puntuación obtenida en cada dimensión de la satisfacción

\begin{tabular}{|lll|}
\hline & Media E.T. & Mediana \\
\hline Exceso trabajo & $2,55-0,035$ & 2,50 \\
\hline Promoción profesional & $2,42-0,038$ & 2,50 \\
\hline Satisfacción laboral (dim.) & $3,15-0,026$ & 3,20 \\
\hline Monotonía laboral & $3,17-0,032$ & 3,25 \\
\hline Relaciones entre profesionales & $3,61-0,034$ & 3,50 \\
\hline Competencia profesional & $4,01-0,058$ & 4,00 \\
\hline Tensión laboral & $2,30-0,041$ & 2,00 \\
\hline Relaciones con compañeros & $3,36-0,025$ & 3,33 \\
\hline Total escala satisfacción laboral & $3,12-0,017$ & 3,15 \\
\hline
\end{tabular}

E.T.: error típico.

Tabla 3

Dimensiones de la satisfacción según categorías profesionales

\begin{tabular}{|l|c|c|c|c|c|c|c|c|}
\hline & \multicolumn{2}{|c|}{ Facultativos } & \multicolumn{2}{c|}{ Enfermeria } & \multicolumn{2}{c|}{ P. no sanitario } & \multicolumn{2}{c|}{ Empresas } \\
& MEDIA & E.T. & MEDIA & E.T. & MEDIA & E.T. & MEDIA & E.T. \\
\hline Exceso de trabajo* & 2,36 & 0,06 & 2,74 & 0,059 & 2,52 & 0,060 & 2,31 & 0,12 \\
\hline Promoción profesional* & 2,40 & 0,064 & 2,54 & 0,062 & 2,26 & 0,066 & 2,76 & 0,21 \\
\hline Satisfacción laboral* & 3,24 & 0,045 & 3,24 & 0,042 & 2,95 & 0,042 & 2,89 & 0,21 \\
\hline Monotonía laboral* $^{*}$ & 3,29 & 0,051 & 3,29 & 0,051 & 2,97 & 0,061 & 2,73 & 0,12 \\
\hline Relaciones entre profesionales & 3,62 & 0,056 & 3,58 & 0,058 & 3,64 & 0,060 & 3,80 & 0,17 \\
\hline Competencia profesional* $^{*}$ & 3,95 & 0,042 & 3,93 & 0,046 & 4,16 & 0,042 & 4,00 & 0,14 \\
\hline Tensión laboral* & 2,05 & 0,064 & 2,55 & 0,070 & 2,28 & 0,069 & 2,41 & 0,17 \\
\hline Relaciones con compañeros* & 3,22 & 0,038 & 3,33 & 0,044 & 3,55 & 0,042 & 3,73 & 0,10 \\
\hline Total satisfacción* & 3,08 & 0,029 & 3,20 & 0,029 & 3,08 & 0,030 & 3,03 & 0,10 \\
\hline
\end{tabular}

E.T: error típico.

* $p<0,01$. Significación calculada mediante métodos no paramétricos 
Tabla 4

Dimensiones de la satisfacción según lugar de trabajo

\begin{tabular}{|l|c|c|c|c|}
\hline & \multicolumn{2}{|c|}{ Equipo de Atención Primaria } & \multicolumn{2}{c|}{ Servicio Urgencias } \\
\hline & MEDIA & E. T. & MEDIA & E. T. \\
\hline Exceso de trabajo & 2,52 & 0,038 & 2,75 & 0,10 \\
\hline Promoción profesiona* & 2,46 & 0,040 & 2,02 & 0,15 \\
\hline Satisfacción laboral* & 3,2 & 0,027 & 2,88 & 0,095 \\
\hline Monotonía laboral & 3,17 & 0,034 & 3,10 & 0,11 \\
\hline Relaciones entre profesionales & 3,63 & 0,035 & 3,76 & 0,11 \\
\hline Competencia profesional & 4,00 & 0,028 & 4,08 & 0,088 \\
\hline Tensión laboral & 2,30 & 0,043 & 2,13 & 0,14 \\
\hline Relaciones con compañeros & 3,36 & 0,028 & 3,55 & 0,055 \\
\hline Total satisfacción & 3,13 & 0,018 & 3,09 & 0,053 \\
\hline
\end{tabular}

${ }^{*} \mathrm{p}<0,01$. Significación calculada mediante métodos no paramétricos.

Tabla 5

Dimensiones de la satisfacción según tipo de contrato

\begin{tabular}{|l|c|ccc|c|c|}
\hline & \multicolumn{2}{|c|}{ En propiedad } & \multicolumn{2}{c}{ Interinidad } & \multicolumn{2}{c|}{ Otros } \\
\hline & MEDIA & E. T. & MEDIA & E. T. & MEDIA & E. T. \\
\hline Exceso de trabajo* & 2,63 & 0,052 & 2,38 & 0,052 & 2,65 & 0,084 \\
\hline Promoción profesional & 2,41 & 0,054 & 2,39 & 0,060 & 2,47 & 0,10 \\
\hline Satisfacción laboral & 3,15 & 0,040 & 3,14 & 0,040 & 3,20 & 0,071 \\
\hline Monotonía laboral & 3,11 & 0,048 & 3,24 & 0,050 & 3,25 & 0,065 \\
\hline Relaciones entre profesionales & 3,53 & 0,053 & 3,64 & 0,049 & 3,77 & 0,076 \\
\hline Competencia profesional & 3,97 & 0,039 & 4,07 & 0,038 & 3,96 & 0,068 \\
\hline Tensión laboral* & 2,45 & 0,056 & 2,09 & 0,057 & 2,40 & 0,12 \\
\hline Relaciones con compañeros & 3,31 & 0,039 & 3,38 & 0,035 & 3,55 & 0,058 \\
\hline Total satisfacción & 3,12 & 0,026 & 3,10 & 0,026 & 3,18 & 0,045 \\
\hline
\end{tabular}

E.T.: error típico.

$* p<0,01$. Significación calculada mediante métodos no paramétricos 
$\left.2,46, \mathrm{IC}_{95 \pi_{k}}: 2,38-2,54\right)$ y satisfacción laboral $\left(2,88, \mathrm{IC}_{95 \%}: 2,69-3,07\right.$, frente a $3,20, \mathrm{IC}_{95 \%}$ : $3,15-3,25)$.

Valorando todas la dimensiones según el tipo de contrato (tabla 5) el personal que no tiene plaza en propiedad valora por debajo del resto el exceso de trabajo $(2,38$, $\left.\mathrm{IC}_{95 \%}: 2,28-2,48\right)$ y la tensión relacionada con el mismo $\left(2, .09, \mathrm{IC}_{95 \%}: 1,98-2,20\right)$.

\section{DISCUSIÓN}

El índice de respuesta obtenido se sitúa en el $80 \%$ y es superior al logrado en otros estudios $^{5,6}$, incluido el realizado en este mismo Área Sanitaria en $1992^{2}$. Esta respuesta no ha sido menor al $75 \%$ en ninguna categoría profesional, por lo que creemos que está suficientemente representada la población de estudio, esto es, los trabajadores de Atención Primaria del Area 10, La generalización de los resultados a otras Areas Sanitarias sería cuestionable, debido a las diferentes características de las poblaciones que se atienden y a las diferentes presiones asistenciales soportadas. Por otra parte, las categorías profesionales utilizadas pueden ser heterogéneas pero, así como en el medio hospitalario las tareas realizadas por los diferentes estamentos están muy diferenciadas, en Atención Primaria el trabajo que realiza un administrativo y un celador de equipo, por ejemplo, es bastante similar, lo que nos ha conducido a agrupar aquellas más parecidas para facilitar el análisis.

A la vista de los resultados podemos decir que los profesionales que trabajan en Atención Primaria del Área 10 se sienten capacitados para realizar su tarea y, en general, las relaciones, tanto entre las diferentes categorías como entre los compañeros de la misma, son satisfactorias.

Sin embargo la tensión relacionada con el trabajo es la dimensión peor valorada por los participantes. Son los médicos y el per- sonal no sanitario quienes la valoran más negativamente. Como se afirma en otros estudios ${ }^{7,8}$, la tensión laboral es una fuente de estrés. Los motivos fundamentales de esta mala valoración por parte de los facultativos podrían ser la excesiva presión asistencial (42 pacientes/día, de media en nuestro Área) ${ }^{9}$, y la responsabilidad en la toma de decisiones clínicas, por las repercusiones que conllevan los errores cometidos. El personal no sanitario valora sobre todo el exceso de trabajo.

La promoción profesional es otra de las dimensiones peor valoradas, sobre todo por el personal no sanitario $y$, teniendo en cuenta el lugar de trabajo, por los trabajadores de los Servicios de Urgencias. Los profesionales de este último colectivo probablemente son los que tienen menos acceso a la formación continuada, en parte por los cambios de turnos en su jornada laboral y porque la mayor parte de la formación va dirigida a los profesionales de los equipos. Sin embargo, en el estudio realizado en este mismo Área en el año 1992, eran las enfermeras las que valoraban más negativamente esta dimensión. El motivo de este cambio en la valoración puede estar en que, actualmente, la enfermería tiene una formación continuada específica de la función que desempeñan en el centro de salud.

El personal de otras empresas y el personal no sanitario son los que valoran más negativamente la satisfacción en el puesto de trabajo. Esto puede ser debido a que muchos de ellos tienen un nivel formativo superior al que necesitan para el trabajo que desempeñan; por otra parte, sus salarios son más bajos que en el resto de los trabajadores $y$ es un colectivo que recibe pocas gratificaciones de los usuarios y de los jefes. Por otro lado, el personal de otras empresas se rige por unas condiciones de contratación distintas a las del personal del Insalud.

El exceso de trabajo también es una dimensión valorada negativamente, sobre todo por el personal de empresas que tiene mucha 
carga de trabajo y poco personal contratado, y por el colectivo de médicos, siendo entre éstos más llamativo en los interinos. La enfermería valora algo mejor que sus compañeros esta dimensión; esto se podría explicar en parte porque a su consulta acuden personas con cita programada y concertada, con lo que pueden decidir en gran medida el número de pacientes que pueden atender diariamente.

Todos los colectivos coinciden en señalar a la competencia profesional como la dimensión mejor valorada. Esto es lógico si tenemos cn cuenta que se trata de un colectivo en general muy cualificado: el $72 \%$ son universitarios, en muchos casos con formación especializada.

La valoración de las dimensiones de satisfacción obtenidas en este estudio coincide con la realizada en el año 1992, Llama la atención que, a pesar de los cambios de gestión producidos entre 1992 y 1997 (principalmentc la dotación de una mayor autonomía de los centros de salud, con el compromiso de cumplimiento de objetivos anuales, un mayor control del gasto farmacéutico apoyado en la información sobre la prescripción de cada facultativo, y un mayor desarrollo de los Sistemas de información: cartera de servicios, incapacidad temporal, gestión presupuestaria, actividad asistencial, prescripción farmacéutica, docencia, etc.), la satisfacción laboral no se ha modificado en este periodo. Unicamente el exceso de trabajo y la tensión laboral, aunque en el primer estudio también son las dimensiones peor valoradas, en la actualidad tienen unas puntuaciones todavía más bajas, lo que podría ser un reflejo de una mayor exigencia por parte de la empresa y de la población a los profesionales de Atención Primaria (cupos más numerosos y mayor cantidad de servicios asumidos).

Por último, nos permitimos una reflexión producida por la experiencia que nos da nuestro propio trabajo. Creemos que la empresa podría mejorar la valoración del profesional en estas dimensiones con algunas medidas a su disposición:
- La promoción profesional podría mejorarse mediante la participación de los trabajadores en tareas adicionales a la consulta, que obtengan un reconocimiento por parte de la $\mathrm{Di}$ rección. Este reconocimiento podría ser mediante la facilitación de la formación continuada $u$ otras actividades, sin que supongan una sobrecarga a la actividad habitual del trabajador. Por otra parte, los puestos de responsabilidad, tanto directivos como organizativos, deberían definirse y reconocerse mediante incentivos monetarios, además de poderse disminuir la labor asistencial e invertir ese tiempo en realizar la tarea organizativa, aspecto que puede ser preferido por los profesionales al económico.

- La excesiva presión asistencial atendida a lo largo del año, en parte debida al escaso número de suplencias, puede ser una causa de la valoración tan negativa de la dimensión exceso de trabajo. Habría que plantearse las consecuencias de este ahorro de suplencias e intentar que los incentivos económicos conseguidos por los buenos resultados se pudieran emplear en aumentar estas suplencias, lo que posibilitaría una mayor asistencia a cursos y a otras actividades.

- La monotonía laboral se podría combatir a través de la flexibilización de tareas en las unidades administrativas de los centros, y mediante la participación de estos profesionales no sanitarios en tareas organizativas, de control de calidad, etc.

- Por último, el exceso de trabajo, el nivel de atención requerido para realizar el trabajo asistencial y las consecuencias graves a las que conducen los posibles errores repercute en una gran tensión laboral. La empresa debería ofrecer al trabaja- 
dor un apoyo jurídico tanto consultivo como ejecutivo.

\section{BIBLIOGRAFIA}

1. Saturno PJ, Quintana O, Varo J. ¿Qué es la calidad? Tratado de Calidad Asistencial en Atención Primaria. Madrid: Dupont Pharma; 1997.

2. Fernández MI, Villagrasa JR, Gamo MF, Vazquez J, Cruz E, Aguirre MV, Andradas V. Estudio de la satisfacción laboral y sus determinantes en los trabajadores sanitarios de un área de Madrid. Rev Esp Salud Pública 1995:69:487-497.

3. Aranaz J, Mira J. Cuestionario Font Roja. Un instrumento de medida de la satisfacción en el medio hospitalario. Todo Hosp 1988; 52: 63-66.

4. House J, Strecher V, Metzer H, Robbins C. Occupational stress and health among men a women in the Tecumseh Community Health Study. Health Soc Behav 1986; 27: 62-77.

5. Acámer F, López C, López-Torres J. Satisfacción laboral de los profesionales sanitarios en atención primaria. Aten Primaria 1997; 20: 401-407.,

6. Ignacio E, Córdoba JA, Serrano A, Figueroa E, Moreno J, Ignacio JM. Factores asociados a la satisfacción de los profesionales en los hospitales públicos andaluces. Todo Hosp 1997; 141: 7- 12.

7. Cooper CL, Rout U, Faragher B. Mental health, job satisfaction and job stress among general practitioners. Br Med J 1989; 298: 366- 370.

8. Burke JR, Richardsen AM. Sources of satisfaction and stress among canadian physicians. Psychol Rep 1990; 67: 1335- 1344.

9. Gerencia del Área 10 del Insalud. Memoria 1997. Madrid: Área 10 del Insalud; 1998. 